\title{
A Pre-BLAST-DFE Technique for the Downlink of Frequency Selective Fading MIMO Channels ${ }^{1}$
}

\author{
Ruly Lai-U Choi, and Ross D. Murch ${ }^{2}$ \\ Department of Electrical \& Electronic Engineering \\ The Hong Kong University of Science \& Technology \\ Clear Water Bay, Kowloon, \\ Hong Kong \\ Email: eeluchoi@ee.ust.hk and eermurch@ee.ust.hk
}

\begin{abstract}
In this paper, we propose a Pre-BLAST-DFE technique for the downlink of frequency selective fading multi-input multi-output (MIMO) channels to combat multiple access interference (MAI) and inter-symbol interference (ISI). In our technique, we perform MIMO pre-equalization and pre-layered space-time processing at the transmitter or base station with a simplified receiver at the mobile station that requires only limited signal processing. An important application is in the downlink so that a simplified mobile station can be constructed. An expression for the signal-to-noise ratio (SNR) and error probability based on the Gaussian approximation of the output noise term is derived. Performance is investigated by analysis and simulation results. In particular, it is demonstrated that the diversity order of this technique is higher than that of the MIMO OFDM with V-BLAST and MIMO OFDM with linear transmit pre-processing. It is also noticed that this technique performs better at high SNR values.
\end{abstract}

\footnotetext{
${ }^{1}$ This work was supported by the Hong Kong Research Grant Council (HKUST6048/00E).

${ }^{2}$ Corresponding Author. Email: eermurch@ee.ust.hk. Phone: (+852) 2358 7044. Fax: (+852) 23350194.
} 


\section{Introduction}

Wireless multi-input multi-output (MIMO) systems with multiple antennas employed at both the transmitter and receiver have recently gained a lot of attention because of their promising improvement in terms of performance and bandwidth efficiency compared to conventional systems. The V-BLAST (Vertical Bell Laboratories Layered Space-Time) technique was proposed as one method to achieve these advantages [1] in flat fading channels. To deal with frequency selective fading channels, the technique of decisionfeedback-equalization (DFE) was proposed to be concatenated with V-BLAST [2] [3]. This DFE concatenated V-BLAST, however, suffers from high complexity, and is not desirable at the receiver of a portable.

In this paper, we propose a Pre-BLAST-DFE technique for the downlink of frequency selective channels. In this technique, we perform MIMO pre-equalization and pre-layered space-time processing at the transmitter or base station with a simplified receiver at the mobile station that requires only limited signal processing. This provides a potential solution to achieve high capacity with a simple receiver structure in the downlink of a frequency selective fading channel. In addition, we derive an expression for the signal-tonoise ratio (SNR) and error probability based on the Gaussian approximation of the output noise term.

Previous work on transmit MIMO systems with signal pre-processing before transmission and with a simplified receiver structure includes transmit maximum ratio combining (MRC) scheme [4] [5], transmit zero forcing scheme [6] [7], and a filter bank method [8]. In addition, the idea of Pre-BLAST for flat fading channels was suggested by [9], which a variant of the Tomlinson-Harashima precoding scheme [10] [11]. The transmit MRC scheme has to be utilized with other multiple access techniques, such as code division multiple access (CDMA), because the scheme itself cannot deal with the interference. On the other hand, the transmit zero forcing scheme tried to pre-eliminate all the interference at the receiver. Some desired signal power is sacrificed during the preeliminating process and therefore, the receiver SNR (signal to noise ratio) may become very small. In contrast, the filter bank method provides a sub-optimal solution for the problem that maximizes the minimum SINR (signal-to-interference-plus-noise ratio) among all accesses or users. 
Our work is different however in that we consider frequency selective channels (including flat fading as a special case) and incorporate a pre-decision feedback equalization (DFE) structure with a Pre-BLAST approach to give our Pre-BLAST-DFE technique. The same problem has recently also been addressed independently in [12]. Analysis and numerical results are provided and these show that the proposed system can combat interference, including multiple access interference (MAI) and inter-symbol interference (ISI), as well as fading, and therefore achieve high capacity as well as low bit error rate. In particular, performance of the MIMO OFDM with V-BLAST [13] and the MIMO OFDM with linear transmit pre-processing by channel inversion [7] are provided for comparison purposes and these demonstrate that the diversity order of the proposed Pre-BLAST-DFE technique is higher than both the MIMO OFDM with V-BLAST and the MIMO OFDM with linear transmit pre-processing.

Our main contributions are 1) introducing the idea of the Pre-BLAST-DFE technique for the downlink of frequency selective channels; 2) providing the transmit forward and backward filter structures; 3) deriving an expression for the SNR and providing Gaussian approximation for the error probability; and 4) numerically demonstrating the diversity order of the proposed Pre-BLAST-DFE technique through numerical results.

The structure of this paper is as follows. In Section II, the model of a MIMO system is introduced, while the transmit forward and backward filter structures are provided in Section III. Section IV provides some performance analysis, while Section V provides sample numerical results. Finally, Section VI concludes our work.

\section{System Model}

We consider a multi-input multi-output (MIMO) system and its block diagram is shown in Figure 1, where $M$ antennas are located at the transmitter or BS and $K$ antennas are located at the receiver. $K$ data streams are transmitted simultaneously and each receive antenna is used to retrieve a data stream. A complex baseband discrete model is assumed throughout this paper.

At the transmitter, let $\mathbf{b}_{i}$ represent the $K \times 1$ transmit symbol vector at time instant $i T$ with zero mean and unity variance. As shown in Figure 1, a feedback signal vector from a backward spatial-temporal (ST) filter ( B ) is added to it and the $K$ resultant signals 
(denoted by a $K \times 1$ vector $\left.\mathbf{x}_{i}\right)$ go through the bank of $K$ modulo operators $\left(\Gamma_{d_{i}}(\right.$.$) ,$ $i=1, \cdots, K)$, respectively, which are defined by

$$
\Gamma_{d_{i}}(x)=x-d_{i}\left\lfloor\frac{x+\frac{d_{i}}{2}}{d_{i}}\right\rfloor .
$$

where $\lfloor c\rfloor$ represents the largest integer less than or equal to $c$ and $d_{i}$ is the modulus of the $i$-th modulo operator. Note that the modulo operator for a complex value is decomposed into two modulo operators of the in-phase and quadrature components. Also, note that to the modulo operators are used to prevent the transmit power being significantly increased by the feedback filter. In order to satisfy $\Gamma_{d}\left(\Gamma_{d}(x)\right)=x, x$ must be in the range $-\frac{d}{2} \leq x<\frac{d}{2}$. That is, $d$ must be larger than two times the maximum absolute value of $x$. Therefore, for PAM modulation, we can choose $d$ to be equal to the constellation size times the minimum distance between neighboring constellation points of the input. For square QAM, we can choose $d$ to be equal to the square root of constellation size times the minimum distance between neighboring constellation points of the input.

After going through the modulo operations, the $K$ output signals (denoted by a $K \times 1$ vector $\mathbf{s}_{i}$ ) are passed through a power control block ( $\mathbf{P}$ ) and scaled to some desired value (power). Then, the signals are transformed by a matrix $\mathbf{W}$ of dimension $M \times K$, which represents the forward transmit spatial filter, and launched into the MIMO channel.

We assume channel information is known at the BS and the channel is quasi-static for each data block. The antenna elements transmit or receive information through a wireless communication channel, which is here characterized by a multipath or frequency selective fading channel. We utilize the simplified tapped delay line multipath channel model of [14], and assume an arbitrary power delay profile with maximum excess delay $T_{\max }$. Also, we define the number of paths $L=\left\lfloor T_{\max } / T\right\rfloor+1$, where $T$ is the symbol duration. Hence, for a particular channel, say the channel between the $m$-th transmit antenna and the $k$-th receive antenna, the complex low-pass impulse response of the channel can be written as

$$
h_{k, m}(t)=\sum_{l=0}^{L-1} h_{k, m}^{(l)} \delta(t-l T),
$$

where the superscript $l$ refers to the $l$-th path of the channel and subscript $k$ and $m$ refer to the $k$-th user (or $k$-th receive antenna) and the $m$-th transmit antenna, respectively. The coefficient $h_{k, m}^{(l)}$ is zero-mean, circularly symmetric, complex Gaussian distributed random 
gain at the time instant $l T$, and we assume that $\left\{h_{k, m}^{(l)}\right\}$ are uncorrelated for all $k, m$ and $l$. Throughout this paper, the squared gain of each link from one transmit antenna to one receive antenna is normalized. That is, $E\left[\left.\sum_{l=0}^{L-1} h_{k, m}^{(l)}\right|^{2}\right]=1$, where $|\cdot|$ represents the absolute value operation and $E[$.] represents the expectation operation. $\delta(t)$ is the delta function. To represent the channel in matrix form, the channel transfer matrix of the system can be written as a block Toeplitz matrix, which is given by

$$
\mathbf{H}=\left[\begin{array}{cccc}
\mathbf{H}^{(0)} & \mathbf{0} & \cdots & \mathbf{0} \\
\mathbf{H}^{(1)} & \mathbf{H}^{(0)} & \ddots & \vdots \\
\vdots & \mathbf{H}^{(1)} & \ddots & \mathbf{0} \\
\mathbf{H}^{(L-1)} & \vdots & \ddots & \\
\mathbf{0} & \ddots & \vdots &
\end{array}\right]
$$

where $\mathbf{0}$ is a zero matrix and $\mathbf{H}^{(l)}$ represents the $l$-th path channel transfer matrix of the system, which is given by

$$
\mathbf{H}^{(l)}=\left[\begin{array}{cccc}
h_{1,1}^{(l)} & h_{1,2}^{(l)} & \cdots & h_{1, M}^{(l)} \\
h_{2,1}^{(l)} & h_{2,2}^{(l)} & \cdots & h_{2, M}^{(l)} \\
\vdots & \vdots & \ddots & \vdots \\
h_{K, 1}^{(l)} & h_{K, 2}^{(l)} & \cdots & h_{K, M}^{(l)}
\end{array}\right] .
$$

We concatenate the transmit symbol vector $\mathbf{b}_{i}$ into blocks of length $Q$ to provide a block transmit symbol vector as $\mathbf{b}=\left[\begin{array}{llll}\mathbf{b}_{1}{ }^{T} & \mathbf{b}_{2}{ }^{T} & \cdots & \mathbf{b}_{Q}{ }^{T}\end{array}\right]^{T}$, where ${ }^{T}$ denotes transpose operation. Similarly, the block forms of $\mathbf{x}_{i}$ and $\mathbf{s}_{i}$ (output signal of the modulo operators) are $\quad \mathbf{x}=\left[\begin{array}{llll}\mathbf{x}_{1}{ }^{T} & \mathbf{x}_{2}{ }^{T} & \cdots & \mathbf{x}_{Q}{ }^{T}\end{array}\right]^{T}$ and $\mathbf{s}=\left[\begin{array}{lllll}\mathbf{s}_{1}{ }^{T} & \mathbf{s}_{2}{ }^{T} & \cdots & \mathbf{s}_{Q}{ }^{T}\end{array}\right]^{T}$, respectively. Therefore, the received signals at the receiver can be given as

$$
\mathbf{r}=\left[\begin{array}{cccc}
\mathbf{H}^{(0)} \mathbf{W} & \mathbf{0} & \cdots & \mathbf{0} \\
\mathbf{H}^{(1)} \mathbf{W} & \mathbf{H}^{(0)} \mathbf{W} & \ddots & \vdots \\
\vdots & \mathbf{H}^{(1)} \mathbf{W} & \ddots & \mathbf{0} \\
\mathbf{H}^{(L-1)} \mathbf{W} & \vdots & \ddots & \\
\mathbf{0} & \ddots & \vdots &
\end{array}\right] \cdot\left[\begin{array}{cccc}
\mathbf{P} & \mathbf{0} & \cdots & \mathbf{0} \\
\mathbf{0} & \mathbf{P} & \ddots & \vdots \\
\vdots & \ddots & \ddots & \mathbf{0} \\
\mathbf{0} & \cdots & \mathbf{0} & \mathbf{P}
\end{array}\right] \mathbf{s}+\mathbf{n}
$$

where $\mathbf{n}$ is a noise vector, whose elements are independent and identically distributed (i.i.d.), zero-mean, circularly symmetric complex Gaussian random variables with variance $\sigma^{2}=N_{0} / 2$ for the real and imaginary parts. By denoting 


$$
\mathbf{C}=\left[\begin{array}{cccc}
\mathbf{H}^{(0)} \mathbf{W} & \mathbf{0} & \cdots & \mathbf{0} \\
\mathbf{H}^{(1)} \mathbf{W} & \mathbf{H}^{(0)} \mathbf{W} & \ddots & \vdots \\
\vdots & \mathbf{H}^{(1)} \mathbf{W} & \ddots & \mathbf{0} \\
\mathbf{H}^{(L-1)} \mathbf{W} & \vdots & \ddots & \\
\mathbf{0} & \ddots & \vdots &
\end{array}\right]
$$

and

$$
\tilde{\mathbf{P}}=\left[\begin{array}{cccc}
\mathbf{P} & \mathbf{0} & \cdots & \mathbf{0} \\
\mathbf{0} & \mathbf{P} & \ddots & \vdots \\
\vdots & \ddots & \ddots & \mathbf{0} \\
\mathbf{0} & \cdots & \mathbf{0} & \mathbf{P}
\end{array}\right],
$$

we can rewrite (5) as

$$
\mathbf{r}=\mathbf{C} \widetilde{\mathbf{P}}_{\mathbf{s}}+\mathbf{n} .
$$

At the receiver, the same modulo operator as at the transmitter is incorporated at each antenna for recovering the signal. This operator is placed after the automatic gain control (AGC) and before the signal decision. Assume that the AGCs at the receiver are perfectly adjusted, whose function can be thought of as the inverse of the power control ( $\mathbf{P}$ ) in this paper. The signals after AGCs can be given by

$$
\mathbf{y}=\tilde{\mathbf{P}}^{-1} \mathbf{C} \tilde{\mathbf{P}} \mathbf{s}+\tilde{\mathbf{P}}^{-1} \mathbf{n}
$$

where $\widetilde{\mathbf{P}}^{-1}$ represents the inverse of $\tilde{\mathbf{P}}$.

\section{Transmit Forward and Backward Filters}

The objective of our system is to eliminate the MAI and ISI. To perform this, the forward transmit spatial filter, $\mathbf{W}$, is chosen such that $\mathbf{H}^{(0)} \mathbf{W}$ is a lower triangular matrix with unity diagonal elements. In this way, the combining transfer matrix given in (6) becomes a lower triangular matrix with unity diagonal elements, so that feedback function can be utilized to eliminate the interference. The backward spatial-temporal filter, B , is incorporated for the feedback function, which is chosen such that

$$
\mathbf{B}=\left[\begin{array}{llll}
\mathbf{0} & \cdots & \mathbf{0} & \mathbf{I}
\end{array}\right]-\left[\begin{array}{llll}
\mathbf{P}^{-1} \mathbf{H}^{(L-1)} \mathbf{W P} & \cdots & \mathbf{P}^{-1} \mathbf{H}^{(1)} \mathbf{W P} & \mathbf{P}^{-1} \mathbf{H}^{(0)} \mathbf{W P}
\end{array}\right],
$$

where $\mathbf{I}$ is an identity matrix and $\mathbf{0}$ is a $K \times K$ zero matrix. Assuming that at least $L-1$ zero symbol durations are inserted between two successive data blocks, it can be shown that the input vector to the modulo operators can be given by 


$$
\mathbf{x}=\mathbf{b}+\left(\mathbf{I}-\tilde{\mathbf{P}}^{-1} \mathbf{C} \tilde{\mathbf{P}}\right) \mathbf{s}
$$

where the matrix $\left(\mathbf{I}-\widetilde{\mathbf{P}}^{-1} \mathbf{C} \tilde{\mathbf{P}}\right)$ is the effective transform matrix of the backward spatialtemporal filter for the data block. By Using (9) and (11), we can obtain

$$
\mathbf{y}=\mathbf{b}+\widetilde{\mathbf{P}}^{-1} \mathbf{n}+\mathbf{s}-\mathbf{x} .
$$

With the definition given in (1) and letting the decision statistics, which are the output of the modulo operators, be $\mathbf{z}$. We can obtain

$$
\mathbf{z}=\mathbf{b}+\hat{\mathbf{n}},
$$

where $\hat{\mathbf{n}}=\widetilde{\mathbf{P}}^{-1} \mathbf{n}-(\mathbf{x}-\mathbf{s}+\mathbf{y}-\mathbf{z})$. The term $(\mathbf{x}-\mathbf{s}+\mathbf{y}-\mathbf{z})$ captures the effect of modulo operators on the noise vector $\widetilde{\mathbf{P}}^{-1} \mathbf{n}$. Since the probability that an entry of noise vector $\tilde{\mathbf{P}}^{-1} \mathbf{n}$ is larger than $d_{i}(i=1, \cdots K)$ is very small for a normal operating range of signal-tonoise ratio $(S N R)$, the effect of these modulo operators on the noise vector is negligible.

A close observation of (13) shows that the decision statistics are equal to the transmitted data symbol plus a noise term. This implies that the MAI and ISI are eliminated totally in our proposed system.

The disadvantage of this system is an increase of the transmit power because of the modulo operation. Assume that for PAM modulation we choose the modulus to be equal to the constellation size times the minimum distance between neighboring constellation points of the input, while for square QAM we can choose the modulus to be equal to the square root of constellation size times the minimum distance between neighboring constellation points of the input. If all the transmit data streams use the same modulation, the total transmit power of our system for the PAM modulation and the square QAM modulation can be given by

$$
P_{0}=\frac{m}{m-1} \operatorname{trace}\left(\mathbf{W P P}{ }^{H} \mathbf{W}^{H}\right)
$$

where $m$ is the constellation size of a PAM modulation and the square root of the constellation size of a square QAM modulation; trace(.) represents the trace operation and ${ }^{H}$ represents the complex conjugate transpose operation. Notice that the power increases a portion of $\frac{1}{m-1}$ while compared to a linear transmit pre-processing technique with a transmit transform matrix WP, whose total transmit power is equal to trace $\left(\mathbf{W P P} \mathbf{P}^{H} \mathbf{W}^{H}\right)$. This portion of the wasted power decreases as $m$ increases, which shows that our system is suited to the higher constellation modulation situation. 
In (14), the matrix $\mathbf{P}$ can be determined based on the $S N R$ requirement and $\mathbf{W}$ is determined based on the channel matrix $\mathbf{H}^{(0)}$. By using the well-known "QR" factorisation, the channel matrix $\mathbf{H}^{(0)}$ can be decomposed as $\mathbf{H}^{(0)}{ }^{H}=\mathbf{Q R}$, where $\mathbf{Q}$ is a unitary matrix and $\mathbf{R}$ is an upper triangular matrix. The amplitudes of the nonzero entries in the matrix $\mathbf{R}$ are $\chi$-distributed with different degrees of freedom [15], [16]. By denoting the $i$-th diagonal element of $\mathbf{R}$ as $r_{i i}$. It can be shown that

$$
\mathbf{W}=\mathbf{Q A},
$$

where $\mathbf{A}$ is a diagonal matrix with diagonal entries $\left\{\frac{1}{r_{i i}^{H}}, i=1, \cdots K\right\}$. Since $\mathbf{Q}$ is a unitary matrix, by substituting (15) into (14), the total transmit power becomes

$$
P_{0}=\frac{m}{m-1} \operatorname{trace}\left(\mathbf{A P P}{ }^{H} \mathbf{A}^{H}\right)
$$

Notice that the ordering of the receive antennas affects the construction of the channel matrix $\mathbf{H}^{(0)}$ and thus affects the total transmit power given in (16) because $\mathbf{A}$ depends on the structure of $\mathbf{H}^{(0)}$. Since there are $K$ receive antennas in our system, there are totally $K$ ! combinations of different ordering. Therefore, the optimal ordering is the one that minimizes the total transmit power or maximizes the $S N R$ of all users among the $K$ ! different combinations. When the DFE concatenated V-BLAST is utilized in the uplink [2] [3], the optimal ordering in the uplink can be applied in this Pre-BLAST-DFE technique for the downlink as a sub-optimal ordering, so that a global search for the ordering, which requires computing all the $K$ ! different combinations, is avoided.

The validation of the system depends on the existence of the forward transmit spatial filter $\mathbf{W}$. Since $\mathbf{H}^{(0)} \mathbf{W}$ is a lower triangular matrix with unity diagonal elements, the rank of $\mathbf{H}^{(0)} \mathbf{W}$ is $K$. Therefore, a necessary condition for the existence of $\mathbf{W}$ is that the number of transmit antennas is larger than or equal to $K$. That is, $M \geq K$.

Finally, we would like to address the issue of computational complexity, which is composed of two parts, the initialization complexity and the processing complexity. The main initialization complexity of this technique includes computing the forward transmit space filter $\mathbf{W}$ and the backward spatial-temporal filter $\mathbf{B}$. W can be computed by first 'QR' decomposing the channel matrix $\mathbf{H}^{(0)^{H}}$ and then using (15), while $\mathbf{B}$ can be obtained by (10). Since $\mathbf{H}^{(0)}{ }^{H}$ is a $M \times K$ matrix, the complexity of its ' $Q R$ ' factorisation is fairly low. The processing complexity of this technique mainly includes the multiplication of the symbol vector with the forward and backward matrices, which approximately equals to 
$L M$ multiplications per symbol. It should be emphasized that the receiver structure in our technique is simple. Only one modulo operation is required at each received antenna.

\section{Performance Analysis}

In order to have a better understanding of the proposed system, we derive an expression for the $S N R$ and the conditional error probability based on the Gaussian approximation in this section. Such approximation is not always good, but we will show that relatively accurate bit error rate estimates can be obtained in this case.

By denoting the $i$-th diagonal element of $\mathbf{P}$ as $p_{i i}$, the total transmit power given in (16) can be simplified further and is given by

$$
P_{0}=\frac{m}{m-1} \sum_{i=1}^{K} \frac{\left|p_{i i}\right|^{2}}{\left|r_{i i}\right|^{2}} .
$$

From (17), it is easy to show that the larger the amplitude of $r_{i i}$, the less the total transmit power required. Alternatively, for a particular amount of total transmit power, the larger the amplitude of $r_{i i}$, the larger the amplitude of $p_{i i}$ and thus the received SNR is for each data stream.

If the required quality of service (QoS) is the same for all the users, all the diagonal elements of $\mathbf{P}$ are the same. That is, $p_{11}=p_{22}=\cdots=p_{K K}=p$. From (17) and assuming that the effect of the modulo operator on the noise is negligible, we can obtain the SNR for a particular total transmit power and channel, denoted by $\gamma$, which is given by

$$
\gamma=\frac{T|p|^{2}}{N_{0}}=\frac{(m-1) T P_{0}}{m N_{0}}\left(\sum_{i=1}^{K} \frac{1}{\left|r_{i i}\right|^{2}}\right)^{-1},
$$

where $T$ is the symbol duration. Hence, by approximating the noise at the output of the modulo operator as Gaussian random variables, the conditional bit error probability for 4QAM modulation can be given by [14]

$$
\operatorname{Pr}\left(e \mid P_{0}, \mathbf{H}\right)=Q(\sqrt{\gamma})
$$

where $Q($.) represents the standard $Q$-function and $\gamma$ is given in (18).

A close observation of (18) shows that the receive space diversity can be obtained because the gain is averaged among the $K$ subchannels. Finally, note that the term on the right hand side of (18) is bounded as 


$$
\frac{\min \left\{\left|r_{i i}\right|^{2}, i=1, \ldots, K\right\}}{K} \leq\left(\sum_{i=1}^{K} \frac{1}{\left|r_{i i}\right|^{2}}\right)^{-1} \leq \frac{\max \left\{\left|r_{i i}\right|^{2}, i=1, \ldots, K\right\}}{K}
$$

where $\max \{$.$\} and \min \{$.$\} represent the maximum and minimum value of the set,$ respectively. Therefore, the value of the $S N R$ is bounded as

$$
\frac{(m-1) T P_{0}}{m N_{0}} \frac{\min \left\{\left|r_{i i}\right|^{2}, i=1, \ldots, K\right\}}{K} \leq \gamma \leq \frac{(m-1) T P_{0}}{m N_{0}} \frac{\max \left\{\left|r_{i i}\right|^{2}, i=1, \ldots, K\right\}}{K} .
$$

This bound shows that the bit error rate (BER) performance of this system is upper bounded by the term $\min \left\{\left|r_{i i}\right|^{2}, i=1, \ldots, K\right\}$ and lower bounded by the term $\max \left\{\left|r_{i i}\right|^{2}, i=1, \ldots, K\right\}$.

\section{Numerical Results}

In this section, the performance of the proposed system is investigated. In the simulation, 4-QAM modulation is utilized. For simplicity, a 2-ray frequency selective channel model with equal power delay profile and the time delay between the two rays of one symbol is assumed. It is assumed that the channel is quasi-static for each data block. 10,000 independent data blocks, each of which consists of 1000 data, are transmitted with independent channels are used to obtain each BER in the Monte-Carlo simulation, while 10,000 independent channels are used to obtain each BER in the Gaussian approximation simulation with the conditional bit error probability given in (19). We define the average $E_{b} / N_{0}$ as the average transmit energy per bit over the noise variance at the each received antenna.

Comparisons are performed with two techniques which denote here as Pre-ZF-OFDM [7] and OFDM-BLAST [13]. In Pre-ZF-OFDM, OFDM with linear transmit preprocessing by channel inversion (or transmit zero forcing) system, linear transmit preprocessing with channel inversion [7] is performed at the transmitter for each subcarrier with a simplified receiver structure. On the other hand, in OFDM-BLAST, MIMO OFDM with V-BLAST is performed in which a V-BLAST receiver with zero forcing detection [1] is performed for each subcarrier, whose performance is similar to a flat fading V-BLAST system.

Figure 2 provides performance comparisons of the Monte-Carlo simulation and the Gaussian approximation derived in Section IV. Three configurations are shown including the number of transmit antennas ranging from 4 to 6 and the number of receive antennas 
being 4. It can be observed that the Gaussian approximation provides good agreement with the Monte-Carlo simulation. This shows that our assumption that the effect of the modulo operator on the noise is negligible is reasonable. As a result, the performance analysis in Section IV appears relatively accurate.

Figure 3 provides a sample performance comparison between our proposed PreBLAST-DFE system and the Pre-ZF-OFDM system when the number of receive antennas, $K$, equals 4 , and the number of transmit antennas, $M$, varies from 4 to 6 . We can see that the slope of the BER curve of the Pre-BLAST-DFE is larger than that of the Pre-ZFOFDM and it outperforms the Pre-ZF-OFDM after certain value of $E_{b} / N_{0}$. In the case of 4QAM, the loss of $3 \mathrm{~dB}$ compared to the Pre-ZF-OFDM at low SNR is due to the small constellation size $(m=2)$, which according to (14) leads to poor power efficiency. The performance of the Pre-BLAST-DFE is only superior at high SNR because of the higher achieved diversity order compared to the Pre-ZF-OFDM. Note that the Pre-BLAST-DFE has greater advantage in higher constellation modulation situation because the portion of the wasted power decreases as the constellation size increases as discussed in Section III. For instance, there is a gain of $1.76 \mathrm{~dB}$ and $2.43 \mathrm{~dB}$ for $16-\mathrm{QAM}$ and $64-\mathrm{QAM}$ compared to 4-QAM, respectively. Moreover, it can be observed that the significant performance improvement can be achieved when more transmit antennas are used. In terms of complexity the initialization stage of Pre-ZF-OFDM can be much higher than that complexity of the Pre-BLAST-DFE technique as each sub-carrier in Pre-ZF-OFDM requires the computation of channel inversion. On the other hand, the processing complexity of the Pre-ZF-OFDM is approximately equal to $M$ multiplications per symbol in addition to the computational complexity of the $M$ IFFTs at the transmit antennas, which might be simpler than the Pre-BLAST-DFE depending on the number of multipath $L$.

Similar to Figure 3, Figure 4 provides a sample performance comparison between the proposed downlink Pre-BLAST-DFE system and the uplink OFDM-BLAST system when the number of antennas at the MS, $K$, equals 4 , and the number of antennas at the BS, $M$, varies from 4 to 6 . We can see that the slope of the BER curve of the Pre-BLAST-DFE is larger than that of the uplink OFDM-BLAST. This shows that the diversity order of the downlink Pre-BLAST-DFE is higher than that of the uplink OFDM-BLAST. 
In Figure 5, we provide comparison results when the number of receive and transmit antennas are related by $M=K+1$ for $K=4,5,6$. It can be observed that the performance of the Pre-BLAST-DFE system improves when $K$ increases, while the performance of the Pre-ZF-OFDM system remains almost the same for all the three scenarios $(M=5,6,7)$. This demonstrates that the receive space diversity is attained in the Pre-BLAST-DFE system, which is consistent with our analysis in Section IV.

Finally, Figure 6 provides performance comparison for the Pre-BLAST-DFE systems with optimal ordering and suboptimal ordering. As mentioned in Section III, the optimal ordering is obtained by finding the one that minimizes the total transmit power or maximizes the $S N R$ of all users among the $K$ ! different combinations, while the suboptimal ordering is obtained by using the optimal ordering in the uplink. It can be observed that the performance of suboptimal ordering is almost the same as that of the optimal ordering.

\section{Conclusions}

In this paper, we have introduced a Pre-BLAST-DFE technique for the downlink of frequency selective channels, which can be thought of as pre-processing form of a VBLAST and DFE at the transmitter. Signal pre-processing is performed at the transmitter or base station with the receiver at the mobile station having a simplified structure that requires only limited signal processing. An expression for the signal-to-noise ratio (SNR) and error probability based on the Gaussian approximation of the output noise term is derived. Moreover, performance is investigated by analysis and simulations results and they demonstrate that the proposed system can obtain higher diversity order than the MIMO OFDM with V-BLAST and the MIMO OFDM with linear transmit pre-processing by channel inversion.

\section{References}

[1] G. D. Golden, G. J. Foschini, R. A. Valenzuela, and P. W. Wolniansky, "Detection algorithm and initial laboratory results using V-BLAST space-time communication architecture," Electronic Letters, vol. 35, no. 1, pp.14-16, Jan. 1999.

[2] X. Zhu, and R. D. Murch, "MIMO-DFE based BLAST over frequency selective channels," IEEE GLOBECOM 2001, pp. 449-503. 
[3] X. Zhu, and R. D. Murch, "Layered space-time equalization for wireless MIMO systems," to appear in IEEE Transactions on Wireless Communications.

[4] A. F. Naguib, A. Paulraj, and T. Kailath, "Capacity improvement with base-station antenna arrays in cellular CDMA," IEEE Trans. Vehicular Technology, vol. 43, pp. 691-698, Aug. 1994.

[5] R. L. Choi, K. B. Letaief, and R. D. Murch, "MISO CDMA transmission with simplified receiver for wireless communication handsets," IEEE Transactions on Communications, vol. 49, pp.888-898, May 2001.

[6] D. Gerlach, and A. Paulraj, "Adaptive transmitting antenna arrays with feedback," IEEE Signal Processing Letters, vol. 1, pp. 150 -152,Oct. 1994.

[7] P. Vandenameele, S. Thoen, M. Engels, and H. D. Man, "A combined OFDM/SDMA approach for WLAN," VTC'99, pp. 1712-1716.

[8] W. Yang, and G. Xu, "Designing smart antenna downlink weighting vectors based on the filter bank concept," Proc. SPIE Conf. Adv. Signal Processing Alg. Arch. and Impl., San Diego, CA, July 1997.

[9] W. Rhee, and J. M. Cioffi, "A very high data rate multiuser communication system with multiple antennas at base station," http://www.stanford.edu / wonjong, Aug. 2001.

[10] M. Tomlinson, "New automatic equaliser employing modulo arithmetic," Electronics Letters, pp. 138-139, March 1971.

[11] H. Harashima, H. Miyakawa, "Matched-transmission technique for channels with intersymbol interference,” IEEE Trans. Comm., pp. 774-780, Aug. 1972.

[12] R. F. H. Fischer, C. Windpassinger, A. Lampe, J. B. Huber, "MIMO precoding for decentralized receivers," ISIT 2002, Switzerland, June 30-July 5, 2002.

[13] R. J. Piechocki, P. N. Fletcher, A. R. Nix, C. N. Canagarajah, and J. P. McGeehan, "Performance evaluation of BLAST-OFDM enhanced Hiperlan/2 using simulated and measured channel data," Electronics Letters, vol. 37, Aug. 2001, pp. 1137-1139.

[14] J. Proakis, Digital Communications, New York; McGraw-Hill, third edition.

[15] A. Papoulis, Probability, Random Variables, and Stochastic Process, New York, McGraw-Hill Inc., 1991.

[16] W. J. Choi, R. Negi, and J. M. Cioffi, "Combined ML and DFE decoding for the VBLAST System," ICC2000, pp. $1243-1248$. 


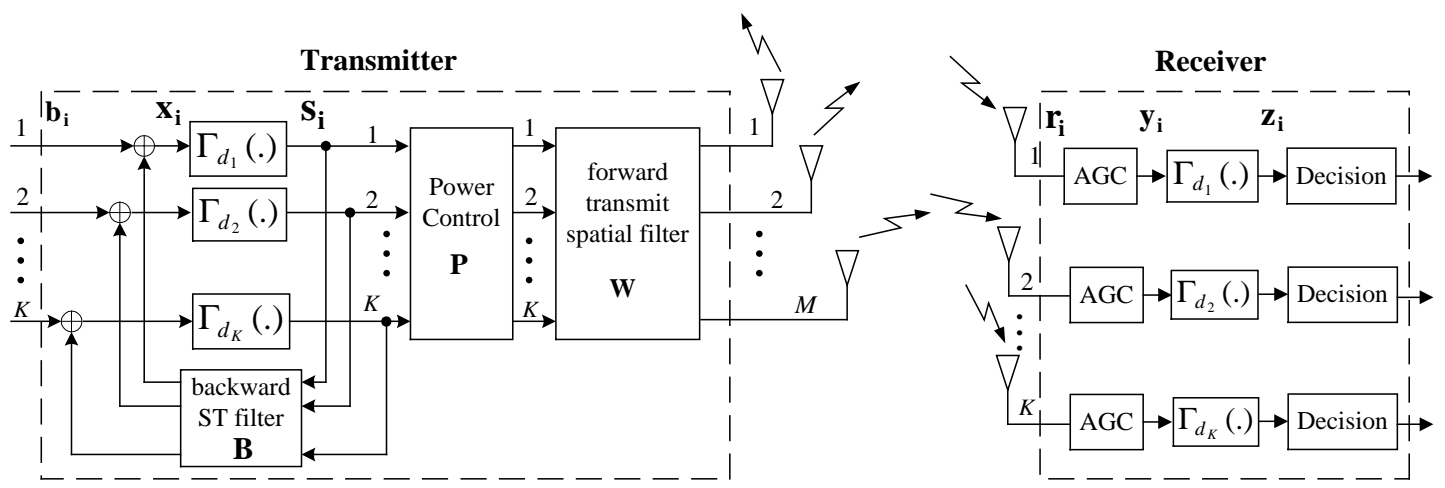

Figure 1. Block diagram of the proposed Pre-BLAST-DFE

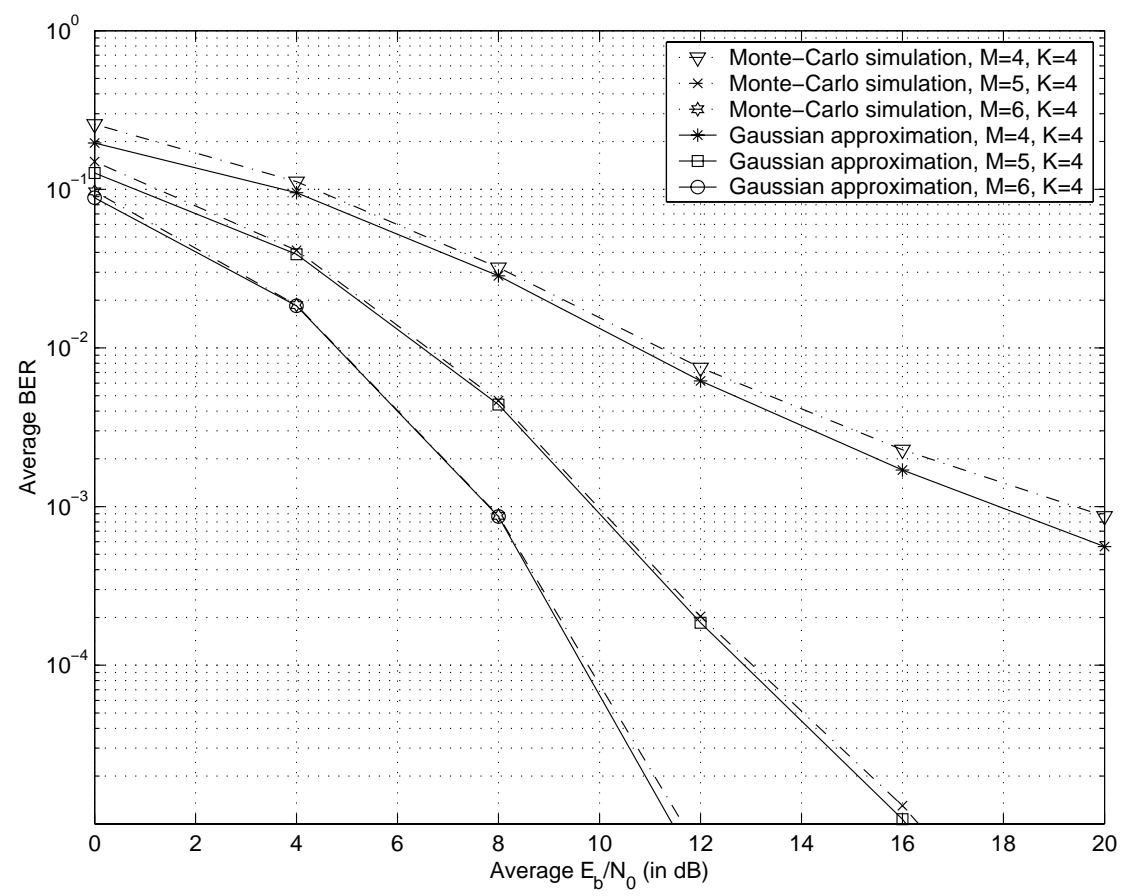

Figure 2. Performance comparisons between Monte-Carlo simulation results and Gaussian approximation simulation results 


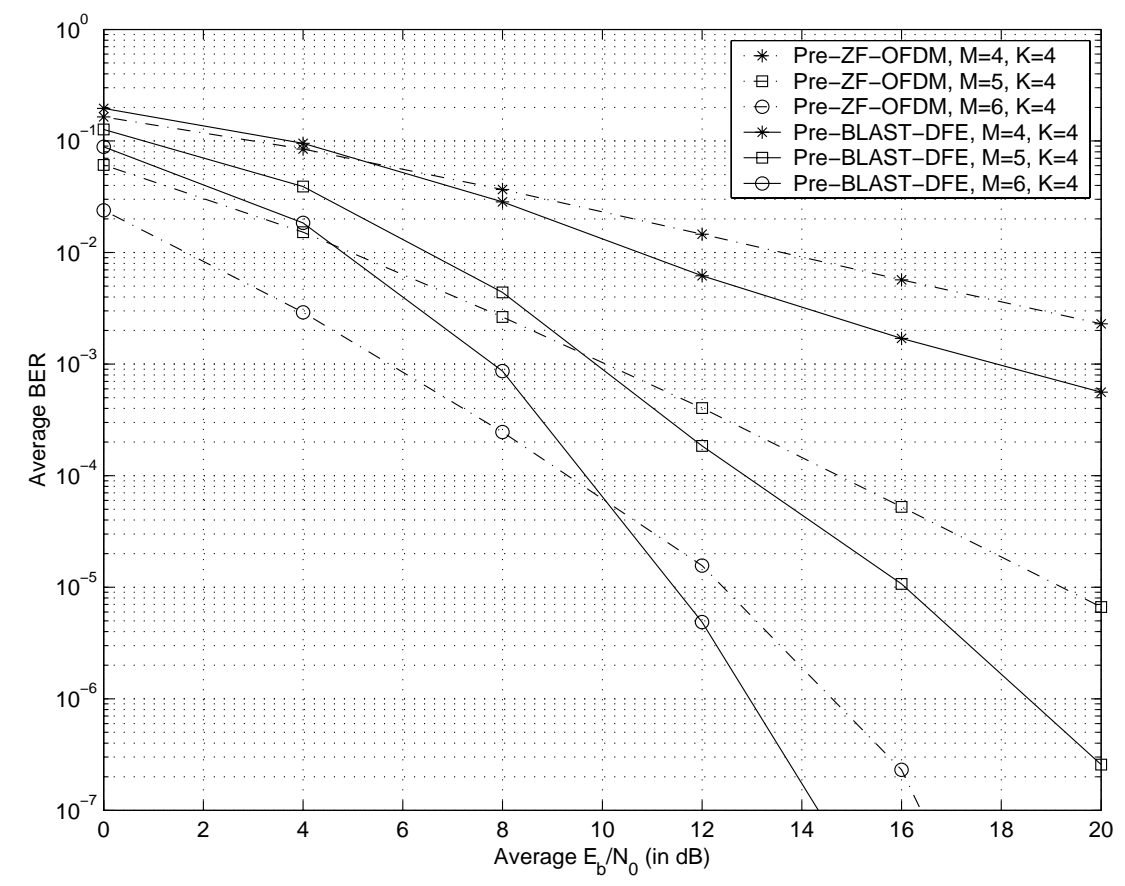

Figure 3. Performance comparison between the Pre-BLAST-DFE system and the Pre-ZFOFDM system when the number of receive antennas, $K$, equals 4

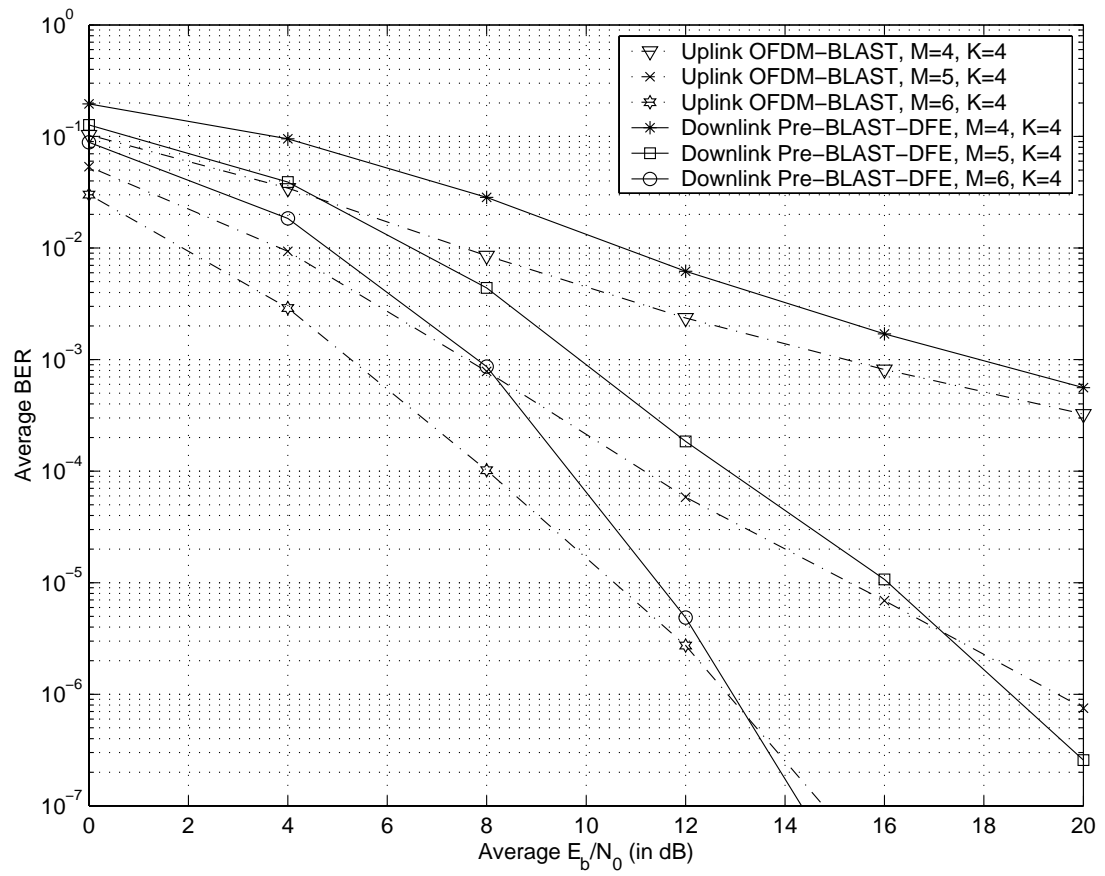

Figure 4. Performance comparisons between uplink OFDM-BLAST and downlink PreBLAST-DFE 


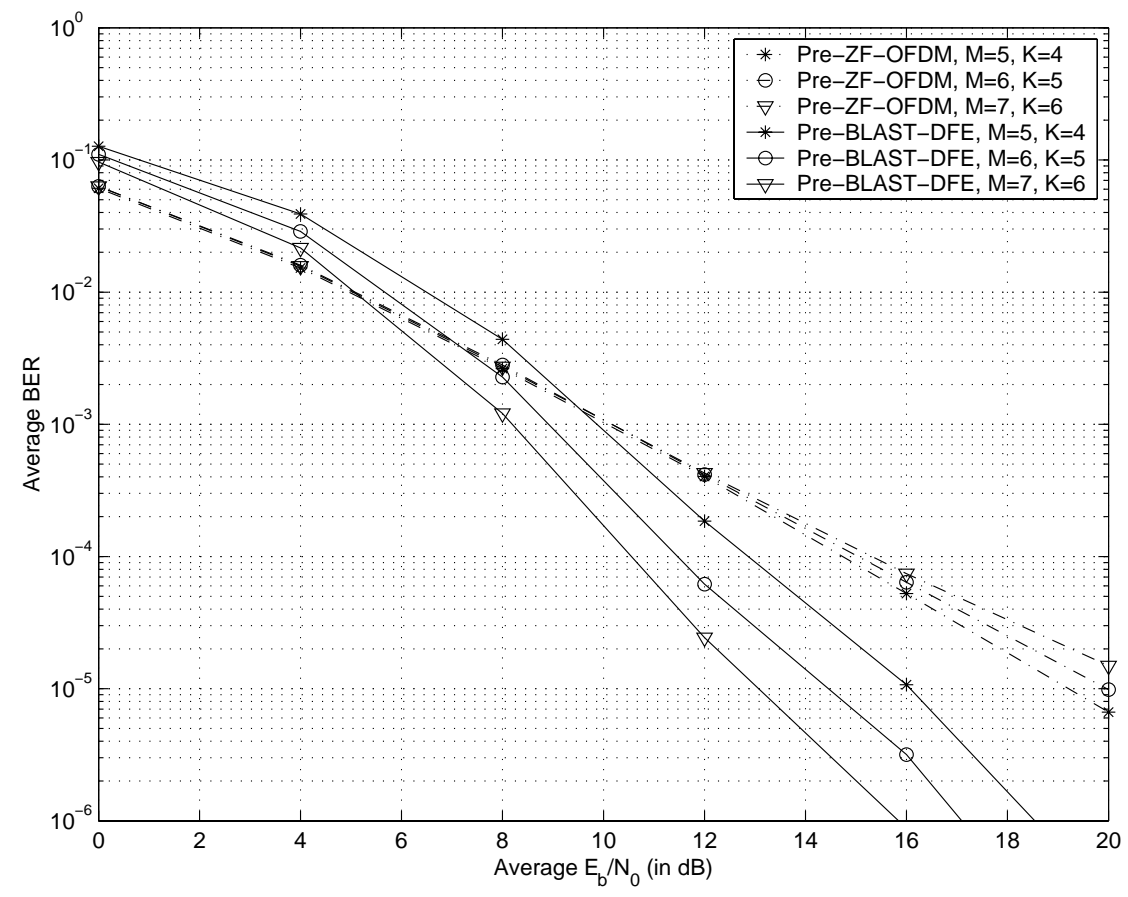

Figure 5. Performance of the Pre-BLAST-DFE system and the Pre-ZF-OFDM system when $M=K+1$

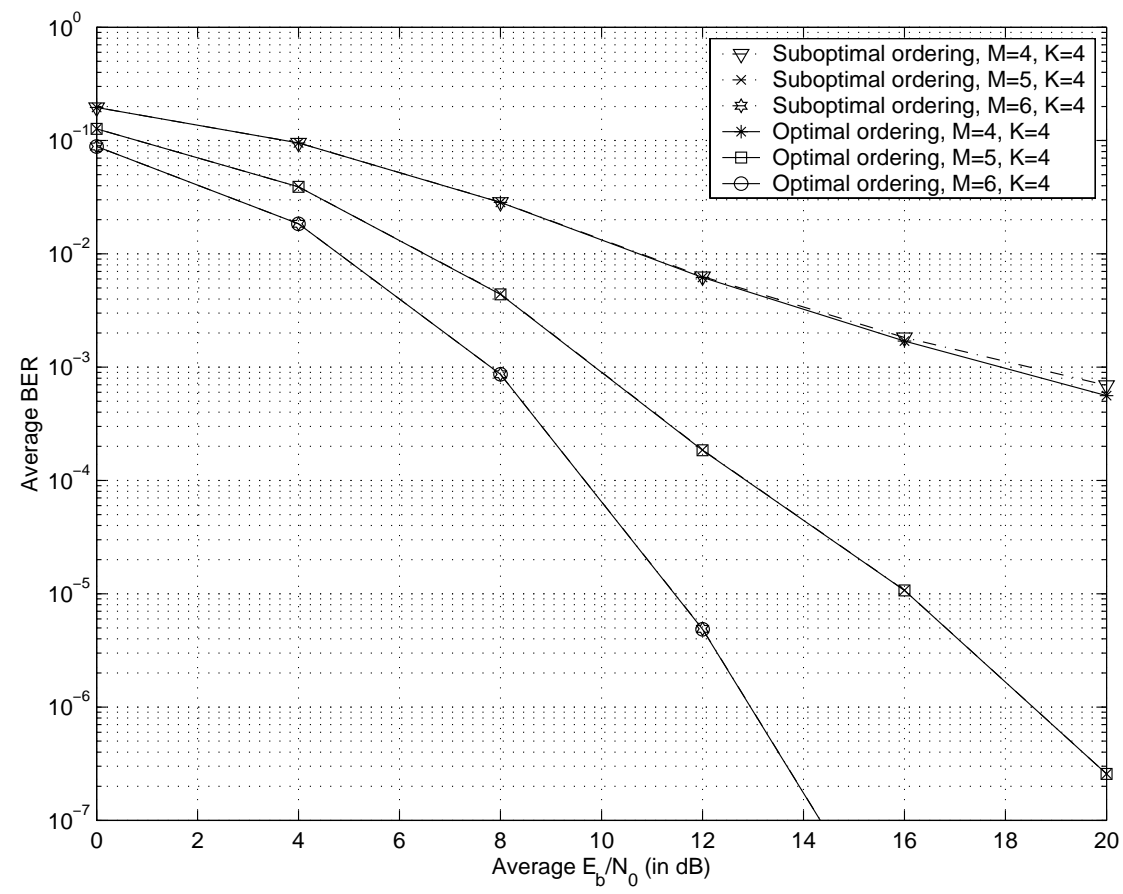

Figure 6. Performance comparisons for Pre-BLAST-DFE systems with optimal ordering and sub-optimal ordering 